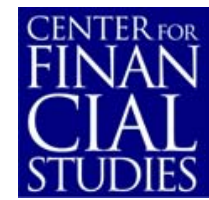

No. $2005 / 14$

Stochastic Optimization and Worst-Case Analysis in Monetary Policy Design

Berc Rustem, Volker Wieland, and Stan Zakovic 


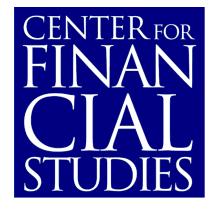

\section{Center for Financial Studies}

The Center for Financial Studies is a nonprofit research organization, supported by an association of more than 120 banks, insurance companies, industrial corporations and public institutions. Established in 1968 and closely affiliated with the University of Frankfurt, it provides a strong link between the financial community and academia.

The CFS Working Paper Series presents the result of scientific research on selected topics in the field of money, banking and finance. The authors were either participants in the Center's Research Fellow Program or members of one of the Center's Research Projects.

If you would like to know more about the Center for Financial Studies, please let us know of your interest.

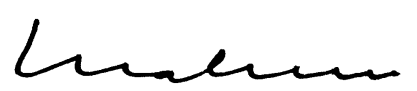

Prof. Dr. Jan Pieter Krahnen

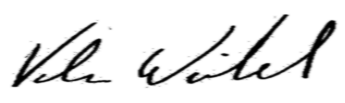

Prof. Volker Wieland, Ph.D. 
CFS Working Paper No. 2005/14

\title{
Stochastic Optimization and Worst-Case Analysis in Monetary Policy Design*
}

\author{
Berc Rustem ${ }^{1}$, Volker Wieland ${ }^{2}$, and Stan Zakovic ${ }^{3}$
}

\section{March 15, 2005}

\begin{abstract}
:
In this paper we compare expected loss minimization to worst-case or minimax analysis in the design of simple Taylor-style rules for monetary policy using a small model estimated for the euro area by Orphanides and Wieland (2000). We find that rules optimized under a minimax objective in the presence of general parameter and shock uncertainty do not imply extreme policy activism. Such rules tend to obey the Brainard principle of cautionary policymaking in much the same way as rules derived by expected loss minimization. Rules derived by means of minimax analysis are effective insurance policies imiting maximum loss over ranges of parameter values to be set by the policy maker. In practice, we propose to set these ranges with an eye towards the cost of such insurance cover in terms of the implied increase in expected inflation variability.
\end{abstract}

JEL Classification: E52, E58, E61

Keywords: Worst-case analysis, robust control, minimax, monetary policy rules, euro area.

\footnotetext{
* We are grateful for helpful comments by conference participants at the SCE Conference on Computing in Economics and Finance 2004 in Amsterdam. Any errors are of course the sole responsibility of the authors.

${ }^{1}$ Department of Computing, Imperial College, London SW7 2BZ, U.K., e-mail: br@doc.ic.ac.uk.

2 Professur fuer Geldtheorie und -politik, Johann-Wolfgang-Goethe Universitaet, Mertonstrasse 17, D-60054 Frankfurt am Main, Germany, tel.: +4969 798-25288, e-mail: wieland@wiwi.uni-frankfurt.de, homepage: http://www.volkerwieland.com.

${ }^{3}$ Department of Computing, Imperial College, London SW7 2BZ, U.K., e-mail: zakovic@doc.ic.ac.uk.
} 


\section{Introduction}

Stochastic optimization remains the principal paradigm for analyzing economic decision making whether it concerns expected utility maximization by households, expected profit maximization by firms or optimal monetary policy design by central banks. As to monetary policy, central bank staff now routinely use macroeconomic models to compute policy rules that aim to minimize expected losses under a variety of economic shocks and parameter values. Such rules form part of regular staff input into central bank decision making. Even members of central bank boards have emphasized implications of expected loss minimization under uncertainty such as Brainard's (1967) prescription of caution in policy making.

Recent research has proposed worst-case analysis - also referred to as minimax or robust control — as an alternative paradigm for economic decision making in general, and monetary policy design in particular. ${ }^{1}$ So far, however, this literature has delivered mixed results concerning the implications of worst-case analysis for the practice of monetary policy. It is criticized for prescribing extreme policy activism and for focusing on very unlikely events (cf. Svensson (2000), Sims (2001)). It is perceived as highly technical and not conducive to practical application.

This paper aims to show that worst-case analysis offers intuitive policy

\footnotetext{
${ }^{1}$ An early advocate of worst-case analysis in monetary policy design was von zur Muehlen (1982). More recent contributions include Sargent (1999), Onatski and Stock (2002), Giannoni (2002), Tetlow and von zur Muehlen (2001) and Zakovic, Rustem and Wieland (2002). For applications in other areas of economic decision making see Hansen and Sargent (2001) and the forthcoming monograph of Hansen and Sargent.
} 
prescriptions and can be of great use for the practice of monetary policy. To this end, we adopt a worst-case robust strategy following Rustem and Howe (2002) and Rustem and Zakovic (2004) rather than the H-infinity approach discussed by Basar and Bernard (1991) and others, and recently applied by contributors to the monetary policy literature. We compare worst-case analysis to expected loss minimization using a small macroeconomic model estimated for the euro area by Orphanides and Wieland (2000). ${ }^{2}$

First, we show that worst-case analysis and expected loss minimization deliver identical policy rules under strict inflation targeting as long as the model used is linear and the uncertainty due to economic shocks is additive and symmetric. In this case, the optimal rule under both approaches is identical to the optimal rule in the absence of shocks, that is, certaintyequivalence applies.

Next, we relax the assumptions of symmetric shocks, known parameters and strict inflation targeting. As to the shocks we show that worst-case analysis provides a straightforward approach for incorporating and insuring against an asymmetric balance of risks. As to the parameters it is well known that uncertainty regarding policy effectiveness, which is a multiplicative parameter ${ }^{3}$, leads to more moderate policy responses under expected loss minimization-Brainard's (1967) principle of cautionary policy. We show that the same principle applies under worst-case analysis in our model. In

\footnotetext{
${ }^{2}$ The model is similar to Svensson (1997) and Ball (1999). Sargent (1999) applied H-infinity robust control to Ball's model.

${ }^{3}$ The respective parameter in our model is the interest-rate sensitivity of aggregate demand.
} 
fact, depending on the range of possible parameter values considered, worstcase analysis may even lead to somewhat more cautious policy responses than expected loss minimization. Flexible inflation targeting, which includes output in the loss function ${ }^{4}$, implies a trade-off between output and inflation. It is well known that this trade-off leads to a more moderate policy response to inflation deviations from target under flexible than under strict inflation targeting. We obtain the same result under worst-case analyis. Quantitatively though, the moderating effect is smaller than under expected loss minimization.

Then, we proceed to consider uncertainty with regard to all parameters in the model. Again we find that Brainard's principle of cautionary policy extends from expected loss minimization to worst-case analysis. Following Orphanides and Wieland (2000) we go beyond the initial linear model and allow for nonlinearity in the inflation-unemployment trade-off. Both algorithms we use in this paper, the mean-variance optimization due to Zakovic et al (2004) and the minimax algorithm due to Rustem and Howe (2002) and Rustem and Zakovic (2004), directly extend to nonlinear models. ${ }^{5}$

Finally, we illustrate the insurance properties of worst-case analysis compared to expected loss minimization. Minimax is shown to provide insurance cover for a given range of shocks and parameter values by establishing the maximum loss over this range. The desired range needs to be specified by

\footnotetext{
${ }^{4}$ The terminology of strict and flexible inflation targeting is adopted from Svensson (1997).

${ }^{5}$ The approach we use for computing optimal response parameters in feedback rules is based on Karakitsos and Rustem (1984) and its extension to robust policies in Rustem (1994).
} 
the policy maker. We obtain a measure of the cost of insurance for a chosen range of shocks and parameters. This cost is best expressed in terms of the deterioration in the expected loss criterion that results from implementing the minimax policy. In practical terms, we propose that an inflation targeting central bank consider the equivalent increase in inflation variability when choosing the range of uncertainties it wants to cover by means of worst-case analysis.

\section{The model}

We use a simple macroeconomic model similiar to Svensson (1997), Ball (1999) and Orphanides (2003). This model has been estimated by Orphanides and Wieland (2000) with annual euro area data from 1976 to 1998. It explains the relationships between three key macroeconomic variables, inflation, $\pi_{t}$, the output gap, $y_{t}$ and the short-term nominal interest rate, $i_{t}$, which is the instrument of monetary policy. For simplicity, the dynamic structure is collapsed to a single lag of output and inflation and inflation expectations are assumed to be backward-looking and equal to past inflation. Under these assumptions, the real interest rate, $r_{t}$, is defined as

$$
r_{t}=i_{t}-\pi_{t}
$$

Output depends positively on its own lag and negatively on the real interest rate according to the following simple aggregate demand equation:

$$
y_{t+1}=\rho y_{t}-\xi r_{t}+u_{t+1} \text {. }
$$


A standard accelerationist Phillips curve relates inflation to lagged inflation and the output gap:

$$
\pi_{t+1}=\pi_{t}+\alpha y_{t+1}+e_{t+1}
$$

The model parameters are $\alpha, \xi>0$ and $\rho \in[0,1)$, while $e_{t+1}, u_{t+1}$ are zeromean normally distributed shocks. In addition, Orphanides and Wieland (2000) consider a nonlinear specification of the Phillips curve:

$$
\pi_{t+1}=\pi_{t}+\alpha_{z} Z\left(y_{t+1} ; \varsigma\right)+e_{t+1} .
$$

The function $Z(y ; \varsigma ; c)$ is approximately zero in a range of width $\varsigma$ and linear outside that range. ${ }^{6}$ An example of such a "zone-linear" Phillips curve is shown in Figure 1. The euro area parameter estimates obtained by Orphanides and Wieland (2000) are summarized in Table 1.

Table 1: Model Estimates

\begin{tabular}{lcc}
\hline \hline$\rho$ & 0.77 & $(0.11)$ \\
$\xi$ & 0.40 & $(0.10)$ \\
$\sigma_{u}$ & 0.84 & \\
$\alpha$ & 0.34 & $(0.13)$ \\
$\sigma_{e}$ & 0.96 & \\
$\alpha_{z}$ & 0.81 & $(0.28)$ \\
$\varsigma$ & 2.0 & \\
$\sigma_{e, z}$ & 0.94 & \\
\hline \hline
\end{tabular}

Values in parenthesis are standard errors. $\sigma_{u}, \sigma_{e}, \sigma_{e, z}$ are standard deviations of shocks.

\footnotetext{
${ }^{6}$ The exact specification of $Z$ used by Orphanides and Wieland is $Z(y ; \varsigma ; c)=y-$ $\frac{1}{2} \sqrt{c+\left(y+\frac{\varsigma}{2}\right)^{2}}+\frac{1}{2} \sqrt{c+\left(-y+\frac{\varsigma}{2}\right)^{2}}$ where $\mathrm{c}$ is set to 0.1 .
} 
Figure 1: Linear and Zone-Linear Phillips Curves

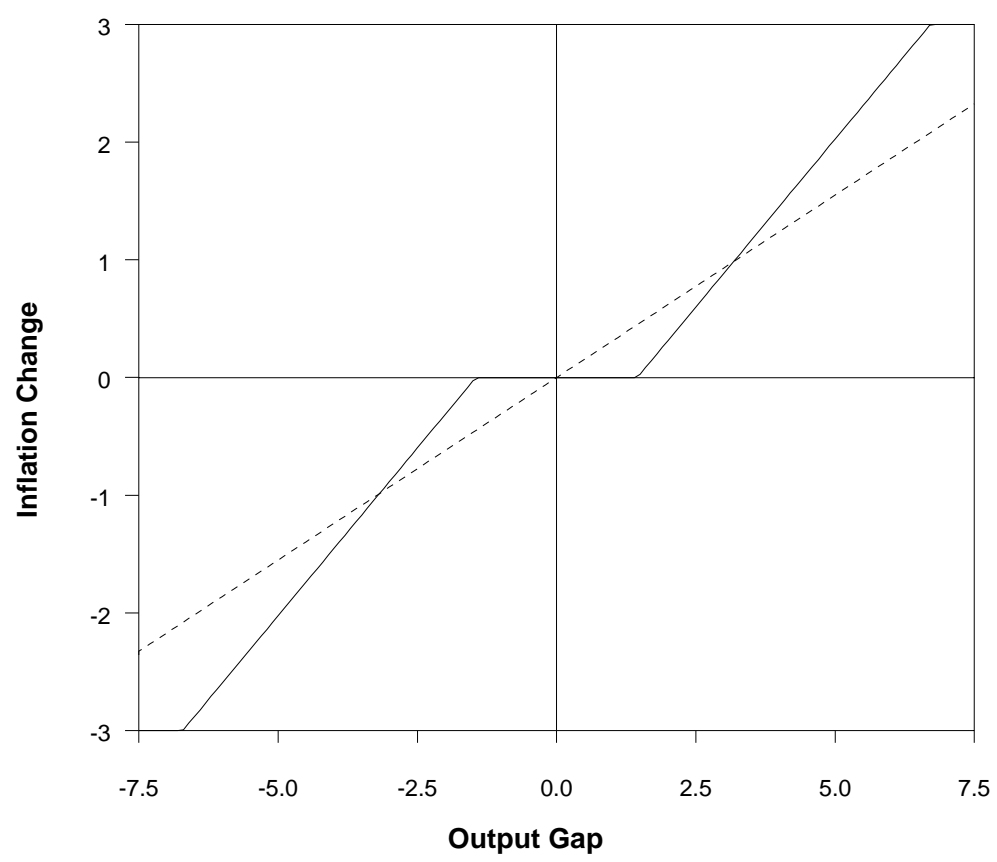

Notes: The dotted line plots the linear Phillips curve $\pi_{t+1}=\pi_{t}+\alpha y_{t+1}$. The solid line plots the zone-linear Phillips curve $\pi_{t+1}=\pi_{t}+\alpha_{z} Z\left(y_{t+1} ; \zeta\right)$ with a slope $\alpha_{z}>\alpha$.

We have chosen this model for its simplicity and its usefulness for organizing our results in terms of key concepts frequently encountered in monetary policy discussions. Even in such a simple model solving for stochastically optimal or minimax policies under general parameter and shock uncertainty remains a nontrivial exercise. The simplicity of the model of course comes at a cost. Specifically, the model is not derived from optimizing household and firm behavior and abstracts from explicit forward-looking modeling of expectations. $^{7}$

\footnotetext{
${ }^{7}$ Thus, we do not take into account the interaction of policy design and private expectations formation, we cannot distinguish between discretion and commitment in policymaking and we cannot address questions of policy credibility. In future work, we plan to extend the stochastic optimization algorithm and the mini-max algorithm used in this
} 
However, we adopt an important lesson from models with forward-looking expectations formation by focusing on the choice of a simple Taylor (1993)style interest rate rule rather than choosing interest rates on a period-byperiod basis. ${ }^{8}$ Thus, the central bank's decision problem consists of choosing the response parameters $x_{\pi}$ and $x_{y}$ in the following rule:

$$
i_{t}=\pi_{t}+x_{\pi}\left(\pi_{t}-\pi^{*}\right)+x_{y} y_{t}
$$

where $\pi^{*}$ denotes the central bank's inflation target. The choice of policy parameters will be made with respect to the central bank's preferences regarding output and inflation performance. We assume a conventional specification of these preferences in form of the following per-period loss function

$$
l_{t+s}=\omega\left(\pi_{t+s}-\pi^{*}\right)^{2}+(1-\omega) y_{t+s}^{2}, \quad \omega \in(0,1) .
$$

Following the terminology of Svensson (1997) $\omega=1$ corresponds to strict inflation targeting and $0<\omega<1$ to flexible inflation targeting.

\section{Stochastic optimization versus worst-case analysis}

Expected loss minimization as of period $t$ implies choosing the parameters of the policy rule, $x_{\pi}$ and $x_{y}$ in equation (5), so as to minimize the expected discounted sum of future per-period losses from $t+1$ onwards subject to the

paper to account for explicit forward-looking expectations formation. A strength of our current algorithms is that they can handle nonlinear specifications of the economy, such as the zone-linear Phillips curve in (4) without difficulty.

${ }^{8}$ See also Levin, Wieland and Williams $(1999,2003)$ for the benefits of simple rules and Tetlow and von zur Muehlen (2001) for an analysis of simple rules under robust control. 
equations determining the dynamics of output, inflation and interest rates:

$$
\min _{x_{\pi}, x_{y}} E_{t}\left[\sum_{s=1}^{\infty} \beta^{t+s-1} l_{t+s}\right] \quad \text { s.t. }(2),(3),(5)
$$

Here $\beta$ denotes the constant discount factor. In forming expectations regarding future losses the central bank needs to consider a wide range of uncertainties. We summarize uncertainty regarding parameters and shocks in the vector $v=[\xi, \rho, \alpha, w]$, where future shocks are collected in the vector $w=\left[\left(u_{1}, e_{1}\right),\left(u_{2}, e_{2}\right), \ldots\right]$. The central bank's objective can then simply be expressed as a function $f(x, v)$ of the vector of policy choices $x$ and the vector of uncertainties $v$ :

$$
f(x, v)=\sum_{s=1}^{\infty} \beta^{t+s-1} l_{t+s}\left(\pi_{t+s}(x, v), y_{t+s}(x, v)\right)
$$

In this notation inflation and output are also expressed as functions of $x$ and $v$, that is $\pi(x, v)$ and $y(x, v)$. These functions are determined by equations (2), (3) and (5). Of course, in the nonlinear version of the model equation (3) will be replaced with equation (4) in defining $\pi(x, v){ }^{9}$

Using the function $f()$ defined by (8) the problem of minimizing expected losses can simply be written as

$$
\min _{x_{\pi}, x_{y}} E_{t, v}(f(x, v))
$$

where $E_{v}$ refers to the expectation with respect to the uncertain variables $v$, which is defined as $v=\bar{v}+\nu$, where $\nu$ is normally distributed with zero mean and standard deviation $\Lambda \sim \mathcal{N}(0, \Lambda)$. Parameter estimates and standard deviations of parameters and shocks are obtained from Table 1.

\footnotetext{
${ }^{9}$ Note also that the nonlinear or zone-linear specification nests the standard linear specification with a zone of zero width $\varsigma=0$.
} 
Compared to expected loss minimization worst-case analysis offers an important advantage. Worst-case analysis does not require any knowledge regarding the probability distributions of unknown parameters and shocks. Instead, the decision maker guards against particular bad outcomes by acting as if he would play a game against nature. In this game nature chooses shocks and parameters to maximize the loss function, while the decision maker chooses the controls to minimize the loss. Thus, following the worstcase approach the central bank in our model chooses $x_{\pi}$ and $x_{y}$ in order to minimise the discounted sum of future per-period losses with respect to the worst possible outcomes of the uncertain shocks $u_{t}$ and $e_{t}$ and parameters $(\alpha, \delta, \rho, \xi)$ that are summarized in the vector of uncertainties, $v$ :

$$
\begin{array}{rl}
\min _{x} \max _{v} & f(x, v), \\
& \text { s.t. } \quad \underline{v} \leq v \leq \bar{v} .
\end{array}
$$

We refer to this minimax approach as box-constrained. $\bar{v}$ and $\underline{v}$ constitute the upper and lower bounds for the uncertain parameters and shocks. These bounds need to bet set by the decision maker. The decision rule derived from (10) then limits the maximum loss over the range defined by these bounds. In other words, this decision rule represents an insurance policy that no worse loss will occur over the set range. Note however, that there may exist multiple global maxima. A question that arises naturally in this context concerns the rationale for setting a specific range. This question is nontrivial when the possible range is infinite. We will provide a practical answer to this question later in this paper. 
$H^{\infty}$ robust control as presented in Basar and Bernhard (1991) constitutes an alternative worst-case approach. Contrary to the minimax approach outline above no interval for the possible value of uncertainties is given. Instead, the $H^{\infty}$ approach solves the transformed minimax problem:

$$
\min _{x} \max _{v} f(x, v)-\gamma^{2}\|v\|_{2}^{2}
$$

where $\gamma=\gamma(\underline{v}, \bar{v})$. The main problem of this approach is the proper choice of $\gamma$. If $\gamma$ is large, it will force $\|v\| \rightarrow 0$. On the other hand, if $\gamma$ is too small it may force $\|v\| \rightarrow \infty$. $H^{\infty}$ seeks the values of $\gamma$ that renders the minimax problem convex in $x$ and concave in $v$. It yields a robust solution that is also a saddle point but does so for a transformed objective.

We now turn to the numerical computation of optimal policies under stochastic optimization and minimax analysis with box constraints. In both cases, we approximate the infinite horizon objective function with a long but finite horizon of 20 periods. The discount factor is set to $\beta=0.9$ and the central bank's inflation target to $\pi^{*}=0$. The parameter values and standard deviations are set as reported in Table $\mathbf{1}$ for the annual euro area model of Orphanides and Wieland (2000). We will consider the linear as well as the nonlinear specification of the economy. For detailed descriptions of the nonlinear stochastic optimization and minimax algorithms we refer the reader to Zakovic et al. (2004) and Rustem and Zakovic (2004) respectively. 


\section{Optimal policy rules under stochastic opti- mization and worst-case analysis}

\subsection{A benchmark case}

First, we consider a benchmark specification, for which expected loss minimization and minimax analysis with box constraints imply identical optimal policy rules. This benchmark corresponds to the linear-quadratic decision problem with a single target variable, known parameters and symmetric shocks. In other words, our benchmark case is a strict inflation targeting central bank, $(\omega=1)$, which only faces uncertainty regarding the additive and symmetric shocks, $u$ and $e$, in the linear model defined by equations (2), (3) and (5). By symmetry of the shocks we mean that expected loss minimization is conducted with respect to normal distributions that are symmetric around a zero mean, while the minimax problem with box constraints is solved with upper and lower bounds that are symmetric around zero.

The solution under expected loss minimization is very simple and familiar. Being only concerned with inflation the central bank can choose the policy response coefficients, $x_{\pi}$ and $x_{y}$, such that all predictable variation in inflation is offset by movements in the real interest rate and output gap. This is possible because the policy rule (5) contains both pre-determined or state variables of the model, lagged inflation and the lagged output gap. Consequently, this rule nests the optimal policy under discretion. The assumptions under the benchmark case ensure that the solution to the infinite horizon stochastic optimization problem defined by (8) and (9) is identical 
to the solution of the following one-period problem: ${ }^{10}$

$$
\begin{array}{ll}
\min _{x_{\pi}, x_{y}} & E_{t}\left(\pi_{t+1}-\pi^{*}\right)^{2} \\
\text { s.t. } & y_{t+1}=\rho y_{t}-\xi\left(i_{t}-\pi_{t}\right)+u_{t+1}, \\
& \pi_{t+1}=\pi_{t}+\alpha y_{t+1}+e_{t+1} \\
& i_{t}=\pi_{t}+x_{\pi}\left(\pi_{t}-\pi^{*}\right)+x_{y} y_{t} \\
& e \sim \mathcal{N}\left(0, \sigma_{e}\right), u \sim \mathcal{N}\left(0, \sigma_{u}\right)
\end{array}
$$

From this one-period problem it is immediately apparent that the following setting of the policy response parameters

$$
x_{\pi}^{E L M}=\frac{1}{\alpha \xi}=7.35, \quad x_{y}^{E L M}=\frac{\rho}{\xi}=1.93
$$

implies that $\pi_{s}=e_{s}+\alpha u_{s}$ for all periods $s=t+1, t+2, \ldots, \infty$. Thus, as stated above all predictable variation in inflation is offset by policy and actual inflation will follow a purely random process. Furthermore, the optimal rule in the model with additive normally distributed shocks is identical to the optimal rule in the absence of those shocks. Thus, certainty-equivalence applies and the optimal response parameters are independent of the degree of uncertainty.

Given the solution for the benchmark case under expected loss minimization it is perhaps not surprising that the solution under minimiax analysis with symmetric upper and lower bounds on shocks $e$ and $u$ turns out to be identical:

$$
x_{\pi}^{\operatorname{minimax}}=\frac{1}{\alpha \xi}=7.35, \quad x_{y}^{\operatorname{minimax}}=\frac{\rho}{\xi}=1.93
$$

\footnotetext{
${ }^{10}$ This point has also been made by Svensson (1997) in the context of strict inflation targeting.
} 
The minimax problem also exhibits certainty-equivalence. For example, we obtain identical response coefficients when considering symmetric bounds such as $(\underline{u} / \bar{u})=\left(-/+k \sigma_{u}\right)$ and $(\underline{e} / \bar{e})=\left(-/+k \sigma_{e}\right)$ for all values of the factor $k \in\{0.5,1,1.5\} .^{11}$

In the remainder of the paper we generalize the central bank's decision problem and consider additional uncertainties while comparing the resulting policy response coefficients to the above benchmark policy.

\subsection{An asymmetric balance of risks}

Having established certainty-equivalence under the common benchmark of symmetric additive uncertainty our first modification is to consider asymmetries in the uncertainty. While such asymmetry is rarely discussed in academic studies, it is standard fare of monetary policy debates under the term 'balance of risks'. Monetary policy makers often perceive information that may bias their assessment of current uncertainties in one direction or the other.

Including an asymmetric balance of risks in standard stochastic optimization analysis would require specifying probability distributions for the shocks that are biased in one of the directions. Instead, worst-case analysis offers an easier route to guard against the dangers arising from an asymmetric balance of risks. The central bank may simply specify a greater upper or lower bound in the minimax problem with box constraints depending on the direction of the perceived risks. Such an asymmetry will break the certainty-equivalence

\footnotetext{
${ }^{11}$ As reported in Table $1 \sigma_{u}=0.84$ and $\sigma_{e}=0.96$.
} 
result obtained above and modify the required policy response.

We illustrate the effect of an asymmetric balance of risks in the minimax problem with box constraints by comparing three scenarios: (i) symmetric bounds (benchmark), (ii) $\left(\underline{u}=-0.5 \sigma_{u}, \underline{e}=-0.5 \sigma_{e}\right)$ and $\left(\bar{u}=\sigma_{u}, \bar{e}=\sigma_{e}\right)$, (high) and (iii) $\left(\underline{u}=-\sigma_{u}, \underline{e}=-\sigma_{e}\right)$ and $\left(\bar{u}=0.5 \sigma_{u}, \bar{e}=0.5 \sigma_{e}\right)$ (low). In scenario (ii) the greater risk is on the high side, while on the low side in scenario (iii).

Table 2 reports the optimal policy response coefficients under the three scenarios specified above. Our findings confirm that asymmetry of the balance of risks breaks certainty-equivalence. Thus, the policy response depends on the degree of asymmetry. For the scenarios considered, the response coefficients with asymmetric risks are found to be smaller than with symmetric risks.

Table 2: Asymmetric risks

\begin{tabular}{lcc}
\hline \hline minimax & $x_{\pi}$ & $x_{y}$ \\
\hline (i) symmetric risk & 7.35 & 1.93 \\
(ii) high-side risk & 6.02 & 1.75 \\
(iii) low-side risk & 6.44 & 1.77 \\
\hline \hline
\end{tabular}

\subsection{Uncertainty regarding policy effectiveness}

With respect to stochastic optimization it is well known that multiplicative parameter uncertainty breaks the certainty-equivalence principle and introduces caution in decision making. In the monetary policy literature such parameter uncertainty is often referred to as Brainard uncertainty due to the 
original contribution of Brainard (1967). He showed that the degree of policy activism varies inversely with the extent of uncertainty about policy effectiveness. Consequently, the response coefficients $x_{\pi}$ and $x_{y}$ in our model should vary inversely with the standard deviation of the interest-rate sensitivity of aggregate demand, $\xi$. As reported previously the euro area estimate of $\xi$ in Orphanides and Wieland (2000) is equal to 0.40 with a standard deviation $\sigma_{\xi}$ of 0.10 .

In the first two columns of Table 3 we report the optimal policy coefficients under expected loss minimization for three levels of uncertainty regarding the interest rate sensitivity of aggregate demand: (i) half the estimated standard deviation, (ii) the estimated standard deviation, and (iii) 1.5 times the estimated standard deviation.

Table 3: Uncertainty regarding $\xi$

\begin{tabular}{lcccc}
\hline \hline & \multicolumn{2}{c}{ ELM } & \multicolumn{2}{c}{ minimax } \\
& $x_{\pi}$ & $x_{y}$ & $x_{\pi}$ & $x_{y}$ \\
\hline & & & & \\
Benchmark & 7.35 & 1.93 & 7.35 & 1.93 \\
(i) $0.5 \sigma_{\xi}$ & 7.08 & 1.88 & 6.80 & 1.87 \\
(ii) $\sigma_{\xi}$ & 6.55 & 1.84 & 6.62 & 1.74 \\
(iii) $1.5 \sigma_{\xi}$ & 6.01 & 1.82 & 6.33 & 1.64 \\
\hline \hline
\end{tabular}

ELM: expected loss minimization.

The results reported under the heading ELM confirm the Brainard principle. Increasing uncertainty regarding $\xi$ leads to a reduction in the optimal responsiveness of the policy rule to output and inflation fluctuations. The coefficient on inflation declines from 7.35 to 6.01 while the coefficient on the output gap declines from 1.93 to 1.82 . As a consequence, the central bank 
conducts interest rate policy in a more cautious manner.

The third and fourth column of Table 3 show the optimal policy coefficients under minimax analysis with box constraints. We consider three sets of symmetric upper and lower bounds: (i) $(\underline{\xi} / \bar{\xi})=\left(0.40-/+0.5 \sigma_{\xi}\right)$, (ii) $(\underline{\xi} / \bar{\xi})=\left(0.40-/+\sigma_{\xi}\right)$, and (iii) $(\underline{\xi} / \bar{\xi})=\left(0.40-/+1.5 \sigma_{\xi}\right)$.

Our first important finding is that the Brainard principle also applies under minimax with box constraints. Widening the upper and lower bounds symmetrically leads to a reduction in the optimal policy coefficients. The coefficient on inflation declines from 7.35 to 6.33 , while the coefficient on output falls from 1.93 to 1.64 . This finding strikes a different note than recent discussions in the literature which have emphasized that worst-case analysis tends to imply a greater extent of policy activism to guard against particularly bad outcomes (cf. Sargent (1999), Onatksi and Stock (2002)).

A second interesting finding is that the degree of policy cautiousness may be more or less pronounced under minimax with box constraints than under expected loss minimization depending on the range of parameter values considered.

\subsection{Flexible inflation targeting}

If one were to compare the optimal policy rules we have computed so far with estimated rules, it would become immediately apparent that the coefficient on inflation deviations from target is extremely large. We have obtained optimal values of $x_{\pi}$ between 6.0 and 7.5 , because we have focused on a strict inflation targeting central bank, $(\omega=1)$. Such a central bank aims 
to eliminate all predictable variation in inflation in our benchmark case with known parameters and additive symmetric shocks. Predictable variation in output, however, is very large under such a policy. Thus, including output deviations from potential in the central bank loss function appears more relevant from an empirical perspective.

Table 4: Flexible inflation targeting

\begin{tabular}{lcccc}
\hline \hline & \multicolumn{2}{c}{ ELM } & \multicolumn{2}{c}{ minimax } \\
& $x_{\pi}$ & $x_{y}$ & $x_{\pi}$ & $x_{y}$ \\
\hline & & & & \\
Benchmark & 7.35 & 1.93 & 7.35 & 1.93 \\
$\omega=0.5$ & 1.86 & 1.93 & 5.21 & 1.87 \\
\hline \hline
\end{tabular}

ELM: expected loss minimization.

For this reason, we consider a flexible inflation targeting central bank that assigns equal weight to output and inflation in the loss function, (i.e. $\omega=0.5$ in equation (6)). Optimal policy coefficients are reported in Table 4. As expected the optimal value of $x_{\pi}$ under expected loss minimization is much smaller, 1.86 instead of 7.35. The coefficient on inflation also declines under minimax analysis but to a lesser extent than under expected loss minimization. Interestingly, under minimax analysis (with symmetric standard deviation bounds on the shocks) both policy coefficients decline as we move from strict to flexible inflation targeting.

\subsection{Uncertainty regarding all parameters and shocks}

We are now ready to proceed with a comparison of expected loss minimization and minimax analysis for flexible inflation targeting taking into account 
uncertainty regarding all parameters and shocks in our model. First, we conduct this comparison for the linear version of our model, which consists of equations (2), (3) and (5) and then for the nonlinear version where the linear Phillips curve, equation (3), is replaced with the zone-linear Phillips curve defined by equation (4) and previously shown in Figure 1.

\section{Linear model}

The linear model contains three unknown parameters, the interest-rate sensitivity of aggregate demand, $\xi$, the coefficient on the lag of the output gap, $\rho$, and the slope of the accelerationist Phillips curve, $\alpha$. We compute the optimal policy that minimizes expected loss using the euro area parameter estimates and standard errors of Orphanides and Wieland (2000) as reported in Table 1. The optimal values for the response coefficients are somewhat smaller than in the case without parameter uncertainty reported in Table 4:

$$
x_{\pi}^{E L M}=1.73, \quad x_{y}^{E L M}=1.89
$$

Thus, again the Brainard principle survives. Including additional sources of parameter uncertainty decreases the extent of policy activism.

Turning to minimax analysis with box constraints we use standard deviation bounds on the shocks $e$ and $u$ and consider three different scenarios for the bounds on the parameters $\xi, \rho$ and $\alpha$ : (i) half the estimated standard deviation, (ii) the estimated standard deviation, and (iii) 1.5 times the estimated standard deviation. 
Table 5: Linear model - all parameters

\begin{tabular}{lcc}
\hline \hline minimax & $x_{\pi}$ & $x_{y}$ \\
\hline (i) 0.5 standard deviation & 4.62 & 1.81 \\
(ii) 1.0 standard deviation & 3.97 & 1.68 \\
(iii) 1.5 standard deviation & 2.80 & 1.60 \\
\hline \hline
\end{tabular}

Again, we confirm that the Brainard principle applies under minimax analysis with box constraints. As the width of the range on the parameters considered increases, the policy response coefficients decline.

\section{Nonlinear model}

We also compute optimal policy rules for the nonlinear version of our model in order to show that the algorithms we use can easily be applied to study important nonlinearities. ${ }^{12}$ We use the estimated zone width, $\varsigma=$ 2.0, for the zonelinear Phillips curve, equation (4) and consider parameter uncertainty regarding the interest-rate sensitivity of aggregate demand, $\xi$, the coefficient on the lag of the output gap, $\rho$, and the slope of the zonelinear Phillips curve, $\alpha_{z}$. The optimal values of $x_{\pi}$ and $x_{y}$ under expected loss minimization turn out to be smaller than in Table 4:

$$
x_{\pi}^{E L M}=0.51, \quad x_{y}^{E L M}=1.86
$$

In particular, the inflation coefficient declines substantially because of the flat zone in the Phillips curve.

As shown in Table 6 minimax analysis with box constraints continues to obey the Brainard principle. Wider bounds for the unknown parameters

\footnotetext{
${ }^{12}$ To maintain comparability to the preceding analysis, however, we do not consider optimal nonlinear policy rules. For an analysis of nonlinear policies under expected loss minimization the reader is referred to Orphanides and Wieland (2000).
} 
imply smaller policy responses to output and inflation and thus less activist policy. The response coefficients now take values that do not appear unusual or extreme compared to actual interest-rate setting by central banks.

Table 6: Nonlinear model-all parameters

\begin{tabular}{lcc}
\hline \hline minimax & $x_{\pi}$ & $x_{y}$ \\
\hline (i) 0.5 standard deviation & 3.29 & 1.66 \\
(ii) 1.0 standard deviation & 2.77 & 1.45 \\
(iii) 1.5 standard deviation & 2.25 & 1.17 \\
\hline \hline
\end{tabular}

\section{How to frame the use of worst-case analysis in monetary policy design}

Having shown that worst-case analysis delivers intuitive policy implications and does not recommend extreme policy activism, the question nevertheless remains, why central banks should use worst-case analysis and if so, how it should be done. Reasons for considering worst-case analysis are not hard to come by.

First, worst-case analysis does not require any confidence in the central bank's ability to specify probability distributions regarding unknown model parameters and economic shocks. In other words, worst-case analysis constitutes a desirable tool for deriving policy recommendations when faced with Knightian uncertainty that precludes the use of stochastic optimization.

Second, worst-case analysis provides insurance that the performance loss is contained over a pre-specified range of uncertainties. This point is illustrated by Table 7 , which reports the maximum losses for alternative ranges of parameters and shocks in the linear and nonlinear model. These losses 
correspond to the values of the function $f(x, v)$ defined by equation (10) for alternative ranges of the uncertainties summarized in $v$. The policy response coefficients associated with these losses have already been reported in Table 5 and Table 6.

Table 7: Maximum losses

\begin{tabular}{lcccc}
\hline \hline & \multicolumn{2}{c}{ Linear model } & \multicolumn{2}{c}{ Nonlinear model } \\
& minimax & ELM & minimax & ELM \\
\hline & & & & \\
(i) 0.5 st. dev. & 62 & 84 & 44 & 55 \\
(ii) 1.0 st. dev. & 100 & 160 & 66 & 91 \\
(iii) 1.5 st. dev. & 175 & 248 & 122 & 173 \\
\hline \hline
\end{tabular}

To give an example for the linear model, the value of 100 in the first column of row (ii) corresponds to the maximum loss under the minimax policy with values of $\alpha, \xi, \rho, e$ and $u$ within one standard deviation from the mean. The respective policy coefficients are $x_{\pi}=3.97$ and $x_{y}=1.68$. Thus, this rule provides an insurance policy that no combination of parameter and shock values within one standard deviation from the mean would generate a loss greater than 100 .

The maximum loss under expected loss minimization, however, is much larger than under minimax analysis. It is reported at a value of 160 in the second column of row (ii). The associated policy coefficients are $x_{\pi}=1.73$ and $x_{y}=1.89$. Thus, moving from expected loss minimization to worst-case analysis reduces the maximum loss by 37.5 percent.

As shown in Table 7 the maximum loss increases with the range of possible parameter values considered. Furthermore, the policy that minimizes expected loss tends to generate consistently higher maximum losses than the 
minimax policy. Thus, worst-case analysis provides substantial insurance benefits.

The question remains how to set the bounds for the range of uncertainties to be covered by the minimax approach. A simple cost-benefit comparison renders the choice of these ranges straightforward. The insurance benefit consists of the reduction in maximum loss reported in Table 7, while the cost of insurance corresponds to the resulting increase in expected loss under the minimax policy. Thus, the decision maker may simply choose the extent of insurance cover depending on its cost in terms of expected loss. For example, as can be seen from Table 8 the cost of one standard deviation of insurance cover on all parameters and shocks corresponds to an increase in expected loss from 17 to 23 in the linear model ( 25 percent) and from 30 to 51 in the non-linear model (70 percent).

Table 8: Expected losses

\begin{tabular}{lcccc}
\hline \hline & \multicolumn{2}{c}{ Linear model } & \multicolumn{2}{c}{ Nonlinear model } \\
& minimax & ELM & minimax & ELM \\
\hline & & & & \\
1.0 standard deviation & 23 & 17 & 51 & 30 \\
\hline \hline
\end{tabular}

Alternatively, the cost of insurance cover by minimax analysis may be expressed in terms of the implied increase in expected inflation variability. For example, the increase in expected loss from 17 to 23 discussed above is equivalent to an increase in the standard deviation of inflation by 20 basis points over the 20 periods considered in our numerical calculations. Presented with the cost of insurance in these terms, a cautious policy maker may well choose to consider a worst-case type rule as benchmark for interest-rate policy. 


\section{Conclusions}

Using a simple estimated model of the euro area we have shown that minimax analysis with box constraints tends to obey the Brainard principle of cautionary policymaking in much the same way as expected loss minimization. We found that simple Taylor-style policy rules optimized under a minimax objective in the presence of general parameter and shock uncertainty do not imply extreme policy activism. Instead, such rules are effective insurance policies limiting maximum loss over ranges of parameter values to be set by the policy maker.

Thus, we propose to frame the use of box-constrained minimax analysis in practical monetary policy design in the following manner. Central bank staff could compute the increase in inflation variability that is equivalent to the increase in expected loss resulting from minimax policies over alternative ranges for the uncertainties. With these measures of the cost of insurance cover at hand, central bank decision makers could then pick a range that they consider affordable.

So far, a limitation of our analysis is the lack of forward-looking expectation terms in the Phillips curve and aggregate demand equations. In future work, we plan to extend our algorithms to allow for such terms in the model. Then, we will be able to address questions concerning the interaction of policy design and private expectations formation, policy discretion versus commitment and policy credibility. Another project, which we can accomplish using the current version of our algorithms, will be to extend our analysis to con- 
sider simple nonlinear policy rules as in Orphanides and Wieland (2000).

\section{References}

Ball, L., (1999), "Policy rules for open economies,"in, Taylor, J.B. (ed.), Monetary policy rules, NBER and University of Chicago Press, Chicago.

Basar, T. and Bernhard, P., (1991), $H^{\infty}$-Optimal control and related minimax design problems, Birkhauser, Boston.

Brainard, W., (1967), "Uncertainty and the Effectiveness of Policy", American Economic Review, 1967, 57, 411-25.

Giannoni, M., (2002), "Does model uncertainty justify caution ? Robust optimal monetary policy in a forward-looking model ", Macroeconomic Dynamics, 6(1), 111-144.

Hansen, L. and T. Sargent (forthcoming), "Misspecification in recursive macroeconomic theory," Manuscript.

Hansen, L. and Sargent, T. J., and Tallarini, T. D., (1999), "Robust permanent income and pricing", Review of Economic Studies 66, pp. 873-907.

Karakitsos, E. and B. Rustem (1984), "Optimally derived fixed rules and indicators", Journal of Economic Dynamics and Control, 8, 33-64.

Levin, A., Williams, J.C., and V. Wieland, (1999), Robustness of simple monetary policy rules under model uncertainty, in John B. Taylor (ed.), Monetary Policy Rules, NBER and Chicago Press, June 1999.

Levin, A., Williams, J.C., and V. Wieland, (2003), "The performance of forecast-based monetary policy rules under model uncertainty,", American Economic Review, 93 (3), June.

Onatski, A. and J. Stock, (2002), "Robust monetary policy under uncertainty in a small model of the macroeconomy ",Macroeconomic Dynamics, 6(1).

Orphanides, A., (2003), "Monetary Policy Evaluation With Noisy Information", Journal of Monetary Economics, 50(3), 605-631.

Orphanides, A., and V. Wieland, (2000), "Inflation zone targeting", European Economic Review, 44, 1351-1387.

Rustem, B., (1994), "Stochastic and robust control of nonlinear economic systems", European Journal of Operational Research, 73, 304-318.

Rustem, B., and M.A. Howe, (2002), Algorithms for worst-case design with 
applications to risk management, Princeton University Press.

Rustem, B. and S. Zakovic, (2004), An interior point algorithm for continuous minimax problems, working paper, Imperial College.

Sargent, T., (1999), "Discussion of policy rules for open economies,"in, Taylor, J.B. (ed.), Monetary policy rules, NBER and University of Chicago Press, Chicago.

Sims, C. A., (2001), "Pitfalls of a minimax approach to model uncertainty," American Economic Review, 91 (2), 51-54.

Svensson, L., (1997), "Inflation forecast targeting: Implementing and monitoring inflation targets", European Economic Review, 41, 1111-1146.

Svensson, L., (2000), "Robust control made simple", working paper, Princeton University.

Taylor, J. B., (1993), Discretion versus policy rules in practice, CarnegieRochester Conference Series on Public Policy, 39, 195-214.

Tetlow, R. and P. von zur Muehlen, (2001), "Robust monetary policy with misspecified models: Does model uncertainty always call for attenuated policy?",Journal of Economic Dynamics and Control, 25, 911-949.

von zur Muehlen, P., (1982), "Activist vs. Non-Activist Monetary Policy: Optimal Policy Rules under Extreme Uncertainty, Manuscript, Federal Reserve Board, Washington, DC, April 1982.

Zakovic, S., Rustem, B., and V. Wieland, (2004), "Mean variance optimization of non-linear systems and worst-case analysis, working paper.

Zakovic, S., Rustem, B., and V. Wieland, (2002), "A Continuous Minimax Problem and its Application to Inflation Targeting", in G. Zaccour, ed., Decision and Control in Management Science, Kluwer Academic Publishers, 2002. 


\section{CFS Working Paper Series:}

\begin{tabular}{|c|c|c|}
\hline No. & Author(s) & Title \\
\hline $2005 / 05$ & Jan Pieter Krahnen & $\begin{array}{l}\text { Der Handel von Kreditrisiken: Eine neue } \\
\text { Dimension des Kapitalmarktes }\end{array}$ \\
\hline $2005 / 06$ & $\begin{array}{l}\text { Günter Franke } \\
\text { Jan Pieter Krahnen }\end{array}$ & $\begin{array}{l}\text { Default Risk Sharing Between Banks and Markets: } \\
\text { The Contribution of Collateralized Debt } \\
\text { Obligations }\end{array}$ \\
\hline $2005 / 07$ & $\begin{array}{l}\text { Dirk Krueger } \\
\text { Harald Uhlig }\end{array}$ & $\begin{array}{l}\text { Competitive Risk Sharing Contracts with One- } \\
\text { Sided Commitment }\end{array}$ \\
\hline $2005 / 08$ & $\begin{array}{l}\text { Torben G. Andersen } \\
\text { Tim Bollerslev } \\
\text { Peter F. Christoffersen } \\
\text { Francis X. Diebold }\end{array}$ & Volatility Forecasting \\
\hline $2005 / 09$ & $\begin{array}{l}\text { Markus Haas } \\
\text { Stefan Mittnik } \\
\text { Bruce Mizrach }\end{array}$ & $\begin{array}{l}\text { Assessing Central Bank Credibility During the } \\
\text { ERM Crises: Comparing Option and Spot Market- } \\
\text { Based Forecasts }\end{array}$ \\
\hline $2005 / 10$ & $\begin{array}{l}\text { Juan Carlo Conesa } \\
\text { Dirk Krueger }\end{array}$ & $\begin{array}{l}\text { On the Optimal Progressivity of the Income Tax } \\
\text { Code }\end{array}$ \\
\hline $2005 / 11$ & $\begin{array}{l}\text { Markus Haas } \\
\text { Stefan Mittnik } \\
\text { Marc S. Paolella }\end{array}$ & $\begin{array}{l}\text { Modeling and Predicting Market Risk With } \\
\text { Laplace-Gaussian Mixture Distributions }\end{array}$ \\
\hline $2005 / 12$ & $\begin{array}{l}\text { Dirk Krueger } \\
\text { Felix Kubler }\end{array}$ & $\begin{array}{l}\text { Pareto Improving Social Security Reform when } \\
\text { Financial Markets are Incomplete!? }\end{array}$ \\
\hline $2005 / 13$ & $\begin{array}{l}\text { Keith Kuester } \\
\text { VolkerWieland }\end{array}$ & $\begin{array}{l}\text { Insurance Policies for Monetary Policy in the Euro } \\
\text { Area }\end{array}$ \\
\hline $2005 / 14$ & $\begin{array}{l}\text { Berc Rustem } \\
\text { Volker Wieland } \\
\text { Stan Zakovic }\end{array}$ & $\begin{array}{l}\text { Stochastic Optimization and Worst-Case Analysis } \\
\text { in Monetary Policy Design }\end{array}$ \\
\hline
\end{tabular}

